\title{
Syncope associated with pain as the presenting feature of neck malignancy: failure of cardiac pacemaker to prevent attacks in two cases
}

\author{
P F Worth, J C Stevens, F Lasri, S Brew, M M Reilly, C J Mathias, P Rudge
}

J Neurol Neurosurg Psychiatry 2005;76:1301-1303. doi: 10.1136/innp.2004.054510

Two patients are described in whom syncope was the presenting clinical feature of an undiagnosed neck malignancy. Both patients also had attacks associated with paroxysms of severe neck pain. Neither patient responded to cardiac pacing.

$\mathrm{R}$ ecurrent syncope as a complication of recurrent neck malignancy is an uncommon but well documented association. $^{12}$ However, syncope as the presenting feature of previously undiagnosed neck malignancy is exceedingly rare. ${ }^{3-5}$ The exact mechanism of the syncope is uncertain; however, a suitably placed neck mass is presumed to cause irritation of afferent fibres from the carotid sinus and body, travelling first in Hering's nerve and then in the glossopharyngeal nerve. Abnormal excitation of these afferent fibres causes excessive vagal activity. Syncope occurs because of bradycardia or asystole and usually prominent vasodepressor hypotension. The mechanism of syncope is therefore similar to that of idiopathic carotid sinus hypersensitivity, which consists of both cardioinhibitory and vasodepressor components in varying proportions.

We describe two patients in whom syncope was the presenting clinical feature of an undiagnosed neck malignancy. Both patients also described attacks associated with paroxysms of severe neck pain.

\section{CASE 1}

A 59 year old man attended his local hospital emergency department having lost consciousness for two minutes after a brief episode of sharp left sided neck pain while driving. Physical examination, chest $x$ ray, and brain computed tomography (CT) were normal. He was discharged home.

He continued to experience occasional left ear pain until two months later, when he had two further blackouts preceded by a similar pain. During the second of these, an ambulance crew reported an unrecordable blood pressure with a pulse rate of 20 beats/min which responded to intravenous atropine.

On admission to hospital, he described a three month history of intermittent left sided tongue numbness, a change in voice quality, dysphagia, and $4 \mathrm{~kg}$ weight loss.

In hospital, he suffered a further episode of sharp neck pain, beginning in the left ear spreading to the left side of the neck and throat, lasting for 30 minutes, when the patient began to feel faint. This was followed by sudden loss of consciousness and urinary incontinence with a blood pressure of $90 / 60 \mathrm{~mm} \mathrm{Hg}$ and pulse 30 beats/min. He recovered consciousness spontaneously after 30 seconds.

A temporary pacing wire was placed but he had a third episode with blood pressure falling to $77 / 40$ despite a satisfactorily paced heart rate. He was subsequently transferred to this hospital. There was no other relevant medical history.

Physical examination revealed a left posterior pharyngeal mass, a left 12th nerve palsy, and a left Horner's syndrome. A dual chamber permanent pacemaker was implanted. However, he suffered two further attacks, with no clear precipitant, during which the pulse rate was normal but blood pressure fell to systolic value of $70 \mathrm{~mm} \mathrm{Hg}$, each time with spontaneous recovery. CT revealed a large mass in the left parapharyngeal region, the upper end of which involved the carotid sheath (fig 1, panels A and B). There was bilateral deep cervical and submental lymphadenopathy. Angiography showed that the internal carotid artery was displaced anteriorly and medially by the tumour and the left internal jugular vein was occluded. There was no abnormal tumour vascularity.

Autonomic function tests after pacemaker insertion revealed no postural hypotension, and neck/head movements did not precipitate hypotension or syncope. Blood pressure responses to isometric exercise, mental arithmetic, cutaneous cold, hyperventilation, and Valsalva manoeuvre were normal. Carotid sinus massage was felt to be unsafe and was not done.

The patient underwent fine needle aspiration followed by Trucut biopsy. The histology was consistent with a high grade B cell lymphoma. He was started on carbamazepine and subsequently received chemotherapy, after which the carbamazepine was discontinued with no further episodes of syncope. He remains in remission from his lymphoma two years later.

\section{CASE 2}

A 71 year old man described an overnight change in his voice, becoming hoarse and strained. He also developed difficulty in swallowing with occasional coughing after swallowing. Two days later he developed a headache affecting the left side of his head, predominantly posteriorly and radiating to his neck. The pain was constant and worse with sitting up but improved over several weeks.

Three months later, he experienced sudden onset of a similar but more severe headache after which he vomited and lost consciousness for approximately two minutes. There were no other prodromal symptoms. He recovered without focal weakness or confusion, and there were no abnormal movements, incontinence, or tongue biting. He was admitted to his local hospital.

On admission, he was in sinus rhythm with a heart rate of 68 beats/min and his blood pressure was 155/70 mm Hg lying and 102/58 standing. Neurological examination was normal.

Abbreviations: CSM, carotid sinus massage; CSS, carotid sinus syndrome 

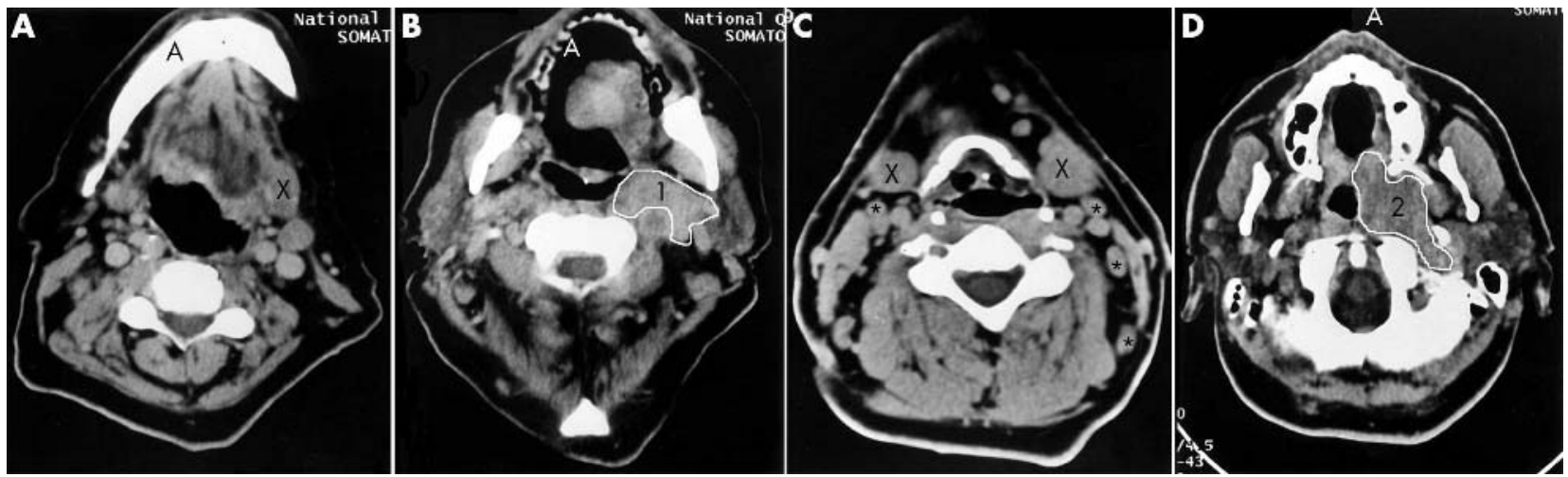

Figure 1 (A) Patient 1. Axial non-contrast computed tomography (CT) of neck showing left deep cervical (" $\left.x^{\prime \prime}\right)$ and bilateral submental lymphadenopathy. (B) More superiorly, there is a large mass (outlined 1) in the left parapharyngeal space, extending posterolaterally to involve the carotid space. (C) Patient 2. Axial post-contrast CT of neck showing bilateral deep cervical lymphadenopathy (" $\left.x{ }^{\prime \prime}\right)$ and scattered smaller nodes ("). (D) There is a heterogeneously enhancing mass occupying the left tonsillar fossa extending anteriorly into the glossotonsillar sulcus and posteriorly to the prevertebral space (outlined 2). The mass extends laterally to involve the carotid space, encasing the left carotid artery and jugular vein.

Shortly after admission, he had one further episode of feeling faint and he collapsed but without loss of consciousness. His blood pressure was recorded as 75/0 and a cardiac monitor showed sinus bradycardia with prolonged pauses up to eight seconds (fig 2) which lasted for seven minutes. A permanent demand pacemaker was fitted. A few hours after the procedure, he had a further collapse with blood pressure unrecordable and pulse of 80 beats/min. CT of the head and neck was normal. He was discharged but had five further collapses without loss of consciousness while sitting or standing, some of which were associated with headache and were preceded by a prodrome of feeling faint. He was admitted to this hospital two months later, six months after the onset of symptoms.

His past medical history included chronic obstructive pulmonary disease, paroxysmal atrial fibrillation, and essential tremor. He was taking aspirin $75 \mathrm{mg}$ daily and hyoscine. He had no relevant family history and had smoked 20 cigarettes a day for 30 years. He had lost $12 \mathrm{~kg}$ over four months.

On physical examination his lying blood pressure was 175/ $77 \mathrm{~mm} \mathrm{Hg}$ and his standing pressure 155/67. Other than the presence of a pacemaker, the cardiovascular, respiratory, and abdominal examination was normal. Neurological examination revealed left 9th, 10th, and 12th nerve palsies. A left sided parapharyngeal mass was visible orally. There was a postural tremor of both upper limbs.

CT of the head and neck showed an enhancing heterogeneous mass occupying the left tonsillar fossa extending

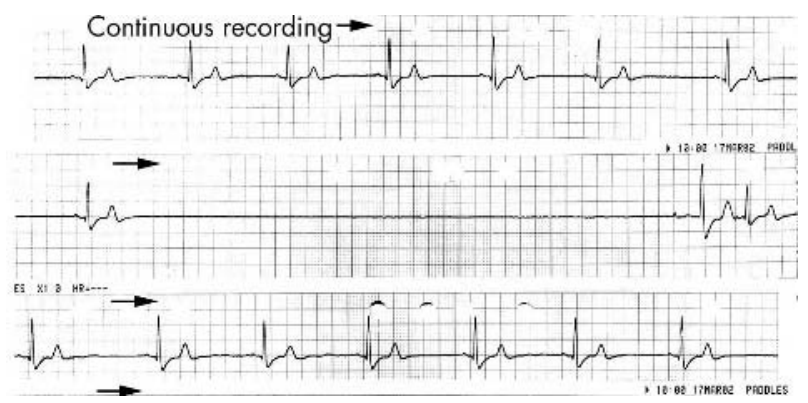

Figure 2 Continuous rhythm strip recording from patient 2 during one of his syncopal events at the referring hospital. Each large square represents 0.2 seconds. The three traces are contiguous. A prolonged sinus pause of approximately 8 seconds is observed, followed by a junctional beat and spontaneous reversion to sinus bradycardia. anteriorly into the glossotonsillar sulcus and bulging into the posterior pharyngeal wall (fig $\mathrm{l}$, panels $\mathrm{C}$ and $\mathrm{D}$ ). The tumour displaced the left carotid artery and jugular vein posterolaterally and appeared to involve these structures. There was bilateral deep cervical lymphadenopathy.

Autonomic function tests revealed postural hypotension, and neck movements failed to induce hypotension or syncope. Carotid sinus massage was not done for safety considerations.

Fine needle aspiration of the mass followed by Trucut biopsy revealed a poorly differentiated squamous cell carcinoma. He was referred to his local radiotherapy service. After radiotherapy, he did not suffer further collapses but died one year after diagnosis.

\section{DISCUSSION}

Syncope as a result of recurrent head and neck cancer has been reported quite often. ${ }^{2-10}$ However, the possibility that syncope could be a presenting feature of an occult tumour is unlikely to be considered by most physicians, especially in the absence of other signs related to local tumour infiltration. Very few cases of this association have been reported..$^{3-5}$ In our case 2, the patient had no focal neurological signs at first presentation and the initial CT of the neck was normal. Both cases had syncope with documented bradycardia/asystole with hypotension, which was not abolished by cardiac pacing. Both were subsequently found to have malignant tumours in the parapharyngeal region. Neither patient had a previous diagnosis of malignancy. Both cases had prominent unilateral head and neck pain.

The exact mechanism of syncope in head and neck malignancy is not well characterised, but is probably similar to that of the carotid sinus syndrome (CSS). In CSS, syncope is painless, often triggered by head movement, and carotid sinus massage (CSM) may be diagnostic. Weiss and Baker described three forms of CSS: the cardioinhibitory type, the vasodepressor type, and the rare cerebral type. ${ }^{11}{ }^{12}$

By contrast, syncope from head and neck cancer usually differs from CSS in several respects. In the case of underlying neck malignancy, while clear triggers may be identifiable, ${ }^{1}{ }^{13}$ the attacks are usually spontaneous and CSM does not characteristically provoke syncopal attacks. ${ }^{14}$ Although excessive vagal tone and consequent cardioinhibition are characteristic of both CSS and malignant syncopal attacks, vasodepressor effects are thought to be much more prominent in the latter. ${ }^{15}$ Deafferentation of the carotid sinus by tumour invasion may account for the inability to provoke 
attacks by CSM in some cases of neck malignancy. It may be that paroxysms of afferent neural activity occur as a result of ephaptic transmission generated in the afferent nerves by the invading tumour.

Both of our cases had cervical lymph node involvement but no direct involvement of the region of the carotid sinus. Therefore, we hypothesise that the combination of pressure on the left carotid sinus and direct injury to or irritation of Hering's nerve or the glossopharyngeal nerve in our cases resulted in prominent vasodepressor effects in association with excessive vagal activity and consequent cardioinhibition. Both our cases failed to respond to insertion of a cardiac pacemaker, with continuing episodes of collapse in association with hypotension. It is self evident that when vasodepressor effects are prominent, whatever the underlying cause, cardiac pacing alone is unlikely to abolish syncope.

Syncope in association with head and neck malignancy

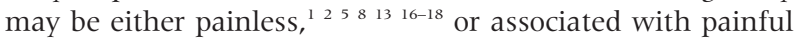
paroxysms which may resemble or be indistinguishable from glossopharyngeal neuralgia. ${ }^{1}$ 4 6-8 101920 The aetiology of glossopharyngeal neuralgia is often idiopathic, but may be secondary to structural abnormalities along the course of the nerve, including neck malignancy. ${ }^{21}$ Rarely, idiopathic glossopharyngeal neuralgia may occur with subsequent syncope. $^{14} 1522-25$

In some patients, carbamazepine ${ }^{1}$ or phenytoin, ${ }^{7}$ or both, has resulted in improvement in pain, but also syncopeprobably because of an effect on afferent neural activity. Some have reported that only surgical section of the intracranial part of the glossopharyngeal nerve is effective in abolishing attacks. ${ }^{20} 23$

We suggest that syncope with bradycardia with or without hypotension should prompt consideration of an occult neck mass. We emphasise the importance of thorough evaluation of the syncopal attacks before permanent cardiac pacing. Attacks which persist despite satisfactory temporary pacing suggest that the mechanism of syncope is likely to include vasodepressor hypotension as well as bradycardia or asystole.

\section{Authors' affiliations \\ P F Worth, J C Stevens, F Lasri, S Brew, M M Reilly, C J Mathias, P Rudge, The National Hospital for Neurology and Neurosurgery, Queen Square, London WCl, UK \\ Competing interests: none declared}

Correspondence to: Dr Paul Worth, Consultant Neurologist, Norfolk and Norwich University Hospital, Colney Lane, Norwich NR4 7UY, UK; paul.worth@nnuh.nhs.uk

Received 20 September 2004

In revised form 17 January 2005

Accepted 28 January 2005

\section{REFERENCES}

1 Macdonald DR, Strong E, Nielsen S, et al. Syncope from head and neck cancer. J Neurooncol 1983;1:257-67.

2 Hong AM, Pressley L, Stevens GN. Carotid sinus syndrome secondary to head and neck malignancy: case report and literature review. Clin Oncol (R Coll Radiol) 2000; 12:409-12.

$3 \mathrm{Kim}$ SS, Lal R, Ruffy R. Bradycardic and vasodepressor syncope secondary to glossopharyngeal neuralgia from hypopharyngeal tumor. Am Heart J 1985;109:1101-2.

4 Weinstein RE, Herec D, Friedman JH. Hypotension due to glossopharyngeal neuralgia. Arch Neurol 1986;43:90-2.

5 Rothstein SG, Jacobs JB, Reede DL. Carotid sinus hypersensitivity secondary to parapharyngeal space carcinoma. Head Neck Surg 1987;9:332-5.

6 Hawkins J, Lewis HD, Emmot W, et al. Vasodepressive carotid sinus hypersensitivity with head and neck malignancy: treatment with propranolol. Am Heart J 1991; 122:234-5.

7 Metheetrairut C, Brown DH. Glossopharyngeal neuralgia and syncope secondary to neck malignancy. J Otolaryngol 1993;22:18-20.

8 Nakahira M, Nakatani H, Takeda T. Syncope as a sign of occult malignant recurrence in the retropharyngeal and parapharyngeal space: $C T$ and MR imaging findings in four cases. Am J Neuroradiol 2002;23:1257-60.

9 Yue AM, Thomas RD. Neurocardiogenic syncope due to recurrent tonsillar carcinoma: successful treatment by dual chamber cardiac pacing with rate hysteresis. Pacing Clin Electrophysiol 2002;25:121-2.

10 Nicholls S, Trim G, Hereford-Ashley P, et al. Pain in the neck. Lancet 2003;361:1700.

11 Weiss S, Baker JR. The carotid sinus reflex in health and disease. Medicine 1933;12:297-351.

12 McIntosh SJ, Lawson J, Kenny RA. Clinical characteristics of vasodepressor, cardioinhibitory, and mixed carotid sinus syndrome in the elderly. Am J Med 1993;95:203-8.

13 Cicogna R, Bonomi FG, Curnis A, et al. Parapharyngeal space lesions syncope-syndrome. A newly proposed reflexogenic cardiovascular syndrome. Eur Heart J 1993; 14:1476-83.

14 Johnston RT, Redding VJ. Glossopharyngeal neuralgia associated with cardiac syncope: long term treatment with permanent pacing and carbamazepine. Br Heart J 1990;64:403-5.

15 Barbash GI, Keren G, Korczyn AD, et al. Mechanisms of syncope in glossopharyngeal neuralgia. Electroencephalogr Clin Neurophysiol 1986;63:231-5.

16 Patel AK, Yap VU, Fields J, et al. Carotid sinus syncope induced by malignant tumors in the neck. Emergence of vasodepressor manifestations following pacemaker therapy. Arch Intern Med 1979;139:1281-4.

17 Almquist A, Gornick C, Benson W, et al. Carotid sinus hypersensitivity: evaluation of the vasodepressor component. Circulation 1985;71:927-36.

18 Holmes FA, Glass JP, Ewer MS, et al. Syncope and hypotension due to carcinoma of the breast metastatic to the carotid sinus. Am J Med 1987;82:1238-42.

19 Sobol SM, Wood BG, Conoyer JM. Glossopharyngeal neuralgia-asystole syndrome secondary to parapharyngeal space lesions. Otolaryngol Head Neck Surg 1982;90:16-19.

20 Dykman TR, Montgomery EB, Gerstenberger PD, et al. Glossopharyngeal neuralgia with syncope secondary to tumor. Treatment and pathophysiology. Am J Med 1981;71:165-70.

21 Giorgi C, Broggi G. Surgical treatment of glossopharyngeal neuralgia and pain from cancer of the nasopharynx. A 20-year experience. J Neurosurg 1984:61:952-5.

22 Rushton JG, Stevens JC, Miller RH. Glossopharyngeal (vagoglossopharyngeal) neuralgia: a study of 217 cases. Arch Neurol 1981;38:201-5.

23 Tsuboi M, Suzuki K, Nagao S, et al. Glossopharyngeal neuralgia with cardiac syncope. A case successfully treated by microvascular decompression. Surg Neurol 1985;24:279-83.

24 Ozenci M, Karaoguz R, Conkbayir C, et al. Glossopharyngeal neuralgia with cardiac syncope treated by glossopharyngeal rhizotomy and microvascular decompression. Europace 2003;5:149-52.

25 Takaya N, Sumiyoshi M, Nakata Y. Prolonged cardiac arrest caused by glossopharyngeal neuralgia. Heart 2003;89:381. 